\title{
Article
}

\section{Can a carer (peer) led psychoeducation programme improve mental health carers well-being, reduce burden and enrich empowerment: a service evaluation study}

Chiocchi, John, Lamph, Gary, Slevin, Paula, Smith, Debrah and Sampson, Mark

Available at http://clok.uclan.ac.uk/25950/

Chiocchi, John, Lamph, Gary ORCID: 0000-0002-4099-2812, Slevin, Paula, Smith, Debrah and Sampson, Mark (2019) Can a carer (peer) led psychoeducation programme improve mental health carers well-being, reduce burden and enrich empowerment: a service evaluation study. Journal of Mental Health Training, Education and Practice, 14 (2). pp. 131-140. ISSN $1755-6228$

It is advisable to refer to the publisher's version if you intend to cite from the work. http://dx.doi.org/10.1108/JMHTEP-10-2018-0057

For more information about UCLan's research in this area go to http://www.uclan.ac.uk/researchgroups/ and search for <name of research Group>.

For information about Research generally at UCLan please go to http://www.uclan.ac.uk/research/

All outputs in CLoK are protected by Intellectual Property Rights law, including Copyright law. Copyright, IPR and Moral Rights for the works on this site are retained by the individual authors and/or other copyright owners. Terms and conditions for use of this material are defined in the policies page. 
Can a carer (peer) led psycho-education programme improve mental health carers wellbeing, reduce burden, \& enrich empowerment 'A service evaluation study'

\begin{tabular}{|r|l|}
\hline Journal: & Journal of Mental Health Training, Education and Practice \\
\hline Manuscript ID & JMHTEP-10-2018-0057.R1 \\
\hline Manuscript Type: & Research Paper \\
\hline Keywords: & $\begin{array}{l}\text { Mental Health, Personality Disorder, Peer Worker, Carer, } \\
\text { Psychoeducation }\end{array}$ \\
\hline \multicolumn{2}{|l}{} \\
\hline
\end{tabular}




\title{
Can a carer (peer) led psychoeducation programme improve mental health carers wellbeing, reduce burden, \& enrich empowerment 'A service evaluation study'
}

\begin{abstract}
Purpose: Carers of people with mental health problems present with high levels of burden, poor mental wellbeing and feelings of disempowerment by mental health services. The aim of this study was to establish whether providing a psychoeducation skills programme for carers would lead to an improvement of mental wellbeing, reduce the levels of burden that carers sometimes feel in caring for someone with mental illness, and also to increase empowerment. This paper provides a service evaluation study of an innovative carer led psychoeducational intervention that was undertaken.
\end{abstract}

Design/ Methodology/ Approach: This programme was initiated and led by a carer who had themselves experienced a lack of service provision to support carers and families in understanding and caring for a relative with severe and enduring mental health diagnoses. A model of co-production was adopted with the carer who led this initiative working closely with an occupational therapist and consultant psychologist in its development and delivery. Data was collected to measure the impact of the training at 5 different time points. The measures employed to measure outcomes were the Warwick- Edinburgh Mental Well-Being Scale (WEMWEBS), the Burden Assessment Scale (BAS) and Family Empowerment Scale (FES).

Findings: Results indicated improved well-being, reduced burden and increased family empowerment in carers who completed this peer led carer initiative psychoeducational programme.

Research Limitations/ Implications: This service evaluation study was conducted in a single site and in the site in which it was developed. The carer consultant who led this evaluation and development of the intervention was also the peer worker who delivered the interventions. Hence, we are unable to ascertain if the results reported are unique to the individual peer worker. The transferability of this programme and generalisability of the result should therefore be treated with caution and further replication of this model and research is required. This would be beneficial to be conducted in an alternative site from where it was developed, delivered by different facilitators and include a control group.

Practical Implications: The evidence from this study indicates that carers are able and willing to attend a group psychoeducational programme. The high number of referrals to the programme in a relatively short timeframe, indicates that there is significant demand for such a service. Implementation of the programme is relatively straight forward. The key challenges for practical implementation are to have the 
right carer to lead and deliver the programme, and the right support system in place for them (financial and supervision). Co-production also is not without challenges, the peer worker and occupational staff need to ensure mutually valued and respected working relationship develop.

Originality Value: This is the first evaluation of the impact of a carer led psychoeducation intervention for carers of people with mental health difficulties in secondary mental health services.

Key Words Mental Health, Personality Disorder, Carers, Peer worker, Psychoeducation.

\section{Paper Type Service Evaluation Study}

\section{Introduction}

The purpose of psychoeducation is to increase knowledge, insight and understanding of mental health and treatment. Psychoeducation for carers of people with mental health difficulties has a growing body of research to support its effectiveness (Cuijpers, 1999; Cassidy et al., 2001). Psychoeducation with carers of people with mental ill health is thought to improve compliance with treatment and reduce relapse (Sin et al., 2017). This project aimed to build on the existing evidence, although it should be noted that there is a dearth of literature sharing carer led initiatives. The aim of the project was to see if a feasible, practical and effective model of a carer led psychoeducation and skills training programme could be delivered successfully to carers of a family member with a variety of mental health diagnosis. Participants additionally had to have family members whom were in contact with secondary mental health services. This service evaluation was conducted in a single site Northwest NHS Trust in England.

This initiative was developed to support and educate carers of a family members who had mental health difficulties including psychosis, personality disorder and who were in treatment with community mental health teams, inpatient hospital admissions or who were frequent attenders to accident and emergency. The principle outcome was to ascertain whether psychoeducation can improve the mental wellbeing of carers and reduce carer burden (McFarlane et al., 2003; 2012). Carers often report feelings of isolation within the healthcare system due to lack of information around diagnosis, financial difficulties, a sense of hopelessness and a system that can stigmatise carers (Buteau, Dawkins, \& Hoffman, 2008).

A structured training programme has been developed by Hoffman \& Fruzetti (2005) called 'Family Connections'. Family Connections is a twelve-week manualised education program for relatives of persons with borderline personality disorder (BPD). The program provided (a) current information and research on mental illness, 
(b) coping skills, (c) family skills, and (d) opportunities to build a support network for family members. Using principles from Dialectical Behaviour Therapy family members are taught to be less judgemental, less critical and enabled to develop more effective interpersonal / communication skills (Gunderson and Hoffman, 2005).

Family interventions for psychosis have demonstrated reduced relapse rates for people with psychosis (Pilling et al., 2002). These programmes involve working with the service user and family together with the focus on improving the interaction and reducing high expressed emotions that can sometimes arise in families where somebody has psychosis (Barrowclough \& Tarrier, 1997).

The programme developed and evaluated here 'Training, Education and Support' (TES) programme was however developed using the principles highlighted in the Family Connections programme (Hoffman \& Fruzzetti, 2005). This training went beyond support and aimed to train carers in skills that enabled them to support family members who have difficulties with managing relationships and emotions more effectively (Gunderson \& Hoffman, 2005). This programme however differs from that of family interventions in psychosis (Barrowclough \& Tarrier, 1997) as it does not include both service user and carers. Having carer only groups enabled peer support networks to develop but also allowed for openness of discussion to raise self-awareness via the development of knowledge and skills. The training package was open to carers, family members of people who have array of mental health needs and was not restricted to those who had a psychosis or borderline personality disorder.

\section{Project background}

This programme was developed by a lived experience carer. The carer initially starting his journey by gaining a seat on the Trust board as a carer representative. This led to the carer being asked to join a team of occupational specialists and lived experience patients in the delivery of the nationally recognised personality disorder training (knowledge and understanding framework, KUF). The KUF training was an innovative co-produced and co-delivered model of training including both occupational experts and lived experience experts. The carer was one of the first carers to be trained nationally to deliver this training. Involvement in the KUF then led to the development of a bespoke locally developed carer intervention called CRISPS (Carers Require Information to Support Personality Symptoms). The educational initiatives described were driven by the development of an educational capabilities framework (National Institute for Mental Health in England NIMHE, 2003a) and the key policy document 'personality disorder, 'no longer a diagnosis of exclusion' (NIMHE, 2003b). The CRISPS programme was a carer led two-session awareness programme. From this training it emerged that the carers and families who took part felt they needed more skills in understanding the difficulties in living with personality disorder. The TES programme we evaluate in this paper was 
therefore subsequently developed. The content of this psychoeducational intervention was led and developed in collaboration with a carer consultant, clinical psychologist and occupational therapist. This co-production is also mirrored in its delivery as the programme was led by a carer consultant and supported by a health professional.

A business case to take this forward, following conversations with commissioners was submitted and subsequently a CQUIN (commissioning for quality and innovation) was agreed to pilot the programme as an intervention. As part of this CQUIN an agreement to audit and review the impact of the programme was agreed. This led to contact with the Trusts Research and Audit Service who following conversation reached the conclusion that this was not research but instead was a service evaluation study, hence ethical approval was not required. The results and overview of the data was shared with commissioners which has led to the carer gaining a substantive NHS position as a 'Highly Specialised Peer Worker'. The data and an analysis of the result are outlined within this paper.

\section{Why a Carer Programme?}

A quality standard for the provision of psychoeducation for carers and families was highlighted in NICE guidance 88 (NICE, 2015). Higher family involvement has been predictive of better outcomes (Mottaghipour \& Bickerton, 2005) and this therefore provided additional support and rationale for the development of this programme. For many patients with mental health difficulties the crisis presentation is triggered by relationship breakdowns. It is not uncommon for family, friends, carers to frequently be themselves feel alone and there is nothing they can do and therefore a psychiatric admission or more input is needed (Motlova et al., 2006). In some case's this may be true but at other times it can be that a lack of understanding and skills at managing people in distress can be facilitating and exacerbating the problem. Carers are in themselves high attenders of health service care with often unexplained medical symptoms and physical health difficulties that are associated with psychological distress and feelings of alienation and distress (Shah, Wadoo \& Latoo, 2010). The aim of this psychoeducation programme was to educate carers hence reducing carer burden, enhancing well-being and empowering carers with greater skill and knowledge.

\section{Why Carer Led?}

In recent years there has been an increased interest in mental health services in the development of peer support roles for those individuals who had lived experience of mental health difficulties. Peer based roles have been more widely used in substance misuse and alcohol services and it is reported that these roles have grown substantially within mental health services (Kilpatrick, Keeney \& McCauley 2017). They explored if these roles were effective or merely tokenistic but cited 
clearly defined role and job specification being necessary in the implementation of this type of intervention to mitigate against this.

The rationale for having a carer led programme was that this could help with the engagement process and also provide the unique lived experience and sharing of what are often encountered complex challenges of being carer. Stigma for carers is still a significant problem and many models of mental health can put the family down as a cause for the person's problems, often not understanding the complex interactions that occur in families of people with mental health needs. Stigma for carers and family members of borderline personality disorder is particularly a problem (Kirtley et al., 2018).

\section{Training Education and Support (TES)}

The co-delivered programme comprised of up to 20, two-hour training sessions. The purpose of this study was to establish if the programme was able to have a positive impact on carer participant needs as measured by burden, well-being and empowerment. Carer participants were offered two educational awareness sessions based on either: Mental Health psychoeducation covering; Schizophrenia, Bi-Polar, Depression and Anxiety or Personality Disorder. Those who had a family member with co-morbidity could attend both awareness sessions. Following this they proceeded to attend five sessions of group-based learning, covering the following; 1. Invalidation 2. Validation 3. Improving Relationships 4. Coping with Emotions and 5. Managing Stressful Situations.

All sessions consisted of a power-point presentation outlining the different elements to be discussed, carers would also complete exercises within the session and also away from the session on their own experience so they can then relate to and understand how their family member experiences difficulties. The carers then progress to the final training and skills element, which is a twelve-module enhancing problem solving skills programme. Outcomes were measured at five different time points, which are indicated below (Table 1).

\section{Table1 - The Training Programme Overview}

Outcome Assessments Time Point 1

Psychoeducation Psychoeducation Session 1

Psychoeducation Session 2

Outcome Assessments Time Point 2

\begin{tabular}{|l|ll|}
\hline \multirow{4}{*}{ Family Skills } & Family Skill 1 & Invalidation \\
\cline { 2 - 3 } & Family Skill 2 & Validation \\
\cline { 2 - 3 } & Family Skill 3 & Improving Relationships \\
\cline { 2 - 3 } & Family Skill 4 & Coping with Emotions \\
\cline { 2 - 3 } & Family Skill 5 & Managing Stressful Situation \\
\hline Outcome Assessment Time Point 3 & \\
\hline Problem Solving & Module 1 & Understanding Mental Health Disorders. \\
\hline
\end{tabular}




\begin{tabular}{|l|ll|}
\hline Sessions & Module 2 & Supporting Recovery from a Mental Health Disorder. \\
\cline { 2 - 3 } & Module 3 & Relapse Prevention. \\
\cline { 2 - 3 } & Module 4 & Manage Symptoms \& Behaviours of Mental Illness. \\
\cline { 2 - 3 } & Module 5 & Communication \& Problem-Solving Skills. \\
\cline { 2 - 3 } & Module 6 & Barriers to Listening. \\
\hline & Outcome & Assessment Time Point 4 \\
\cline { 2 - 3 } & Module 7 & Conflict Situations. \\
\cline { 2 - 3 } & Module 8 & Stages of Change in Behaviour. \\
\cline { 2 - 3 } & Module 9 & Impact of Mental Illness on the Family. \\
\cline { 2 - 3 } & Module10 & Effect of Mental Illness on different family Members. \\
\hline & Module 11 & Setting Boundaries \& Limits. \\
\cline { 2 - 3 } & Module 12 & Enhancing Relationships Within the Family. \\
\hline
\end{tabular}

On completion of the programme carers were offered a point of contact with the peer carer who led the programme, this post programme support was optional.

\section{Method of delivery}

The education and skills components of this programme are delivered by a peer support carer, who has lived experience of caring for a family member with mental health needs and an experienced mental health practitioner delivering interventions and education in group formats. The peer support carer provides ongoing support to carers once the programme has ended and if a relapse re-occurs for six months after the programme. A small number of carers have accessed this support mainly for reassurance in how they are coping with situations.

\section{Inclusion Criteria}

This was primarily for carers of a family member with a mental health diagnosis and who may also experience psychosis or personality disorder amongst others and who are in contact with the secondary mental health services recovery team, assessment team, home treatment team, inpatient care, or mental health liaison. Additionally some carers in the geographical trust footprint were referred by primary care psychotherapist and local carer centres. This ensured that family members whom are less involved in the patients care were not excluded from the opportunity to receive this intervention.

\section{Exclusion Criteria}

Exclusion criteria included any carers under the age of eighteen as this project was project was aligned to adult services. We also excluded involvement of carers if it was deemed to be clinically inappropriate to work with them or if NICE guidance would recommend alternative approaches.

\section{$\underline{\text { Recruitment }}$}


Recruitment and involvement in this study took place between $22^{\text {nd }}$ September 2014 to $26^{\text {th }}$ June 2017 . Carers were identified initially either by their family members engagement within mental health services, primary care psychological therapies services, or local carer centres. They were then offered an appointment with the peer support carer either face to face or telephone to explain what the programme entails.

\section{Methodology}

The carers attending the training completed three assessment questionnaires; these were the Warwick-Edinburgh Mental Well-being Scale (Tennant et al., 2007), The Burden Assessment Scale (Reinhard et al., 1994) and the Family Empowerment Scale (Kageyama et al., 2016) (Table 2).

\section{Table 2 Outcome Measures}

\begin{tabular}{|l|l|}
\hline Outcome Measure's & Description \\
\hline $\begin{array}{l}\text { The Warwick-Edinburgh Mental Well- } \\
\text { being Scale (WEMWBS) }\end{array}$ & $\begin{array}{l}\text { Includes 14 statements relating to } \\
\text { feeling and thoughts }\end{array}$ \\
\hline The Burden Assessment Scale (BAS) & $\begin{array}{l}\text { Includes 19 specific questions to assess } \\
\text { the perceived burden of carers }\end{array}$ \\
\hline Family Empowerment Scale (FEM) & $\begin{array}{l}\text { Includes 27 items /self-report scale for } \\
\text { carers }\end{array}$ \\
\hline
\end{tabular}

Carers were asked to complete the questionnaires at five different time points over the course of the programme (Table 1). The first set of questionnaires were completed before the awareness sessions, hence ascertaining baseline knowledge and understanding of different mental health diagnoses i.e. Schizophrenia, Bi-Polar, Depression, Anxiety, Personality Disorder. The second set of questionnaires were completed after the awareness sessions, hence on completion of the two educational awareness sessions based on either Schizophrenia, Bi-Polar, Depression, Anxiety or Personality Disorder. A further measure was taken on completion of the skills training programme component. Then finally carers completed the questionnaires at the halfway stage and at the end of the problemsolving modules. In total 166 carers were recruited to the programme. Sixty-three did not take a place on the programme and withdrew prior to commencing. Thirty-eight participants partially completed the programme dropping out after the mental health awareness sessions, as the opted as only wanting the awareness sessions, not the full programme. A further five participants only completed some of the programme due to their personal circumstances changing. In total sixty fully completed the programme and are reported within the results of this paper (Table 3 ).

Table 3 - Participant Programme Engagement

\begin{tabular}{|l|r|}
\hline Total Carer Referrals & 166 \\
\hline Carers who did not engage from start & 63 \\
\hline Carers who attended awareness only & 38 \\
\hline
\end{tabular}




\begin{tabular}{|l|c|}
\hline Carers who left due to personal reasons & 5 \\
\hline $\begin{array}{l}\text { Carers who completed the whole } \\
\text { programme }\end{array}$ & 60 \\
\hline
\end{tabular}

In total the results of seven completed groups are reported within this paper. Five of the groups were made up of nine participants, one of eight participants and one of seven participants.

\section{Results}

All sixty treatment completers engaged in the measuring of outcomes. Each of the three scales (Mental Health \& Wellbeing, Burden Assessment and Family Empowerment) were completed by each individual at five different time points throughout the course. Only those who completed the programme $(\mathrm{N}=60)$ were included in this analysis and $70 \%$ were female and $30 \%$ were male. All participants to complete the programme also completed the evaluations at each of the timepoints outlined.

\section{Patient Diagnosis}

The identified diagnoses of the patients whom carers were supporting were varied as can be seen in Table 4. A majority of those who took part identified with their family member having a diagnosis of either borderline personality disorder or bi-polar disorder. A smaller sample identified their family member as having a depression or anxiety related disorder and a very small number identified schizophrenia as being the patient's diagnosis.

\section{$\underline{\text { Table } 4 \text { - Patient Diagnosis }}$}

\begin{tabular}{|l|l|}
\hline Patient diagnosis & $\%$ \\
\hline BPD & 45.0 \\
Depression/anxiety & 13.3 \\
Bi-polar & 31.7 \\
Schizophrenia & 3.3 \\
BPD/anxiety & 5.0 \\
BPD/bi-polar & 1.7 \\
Total & 100 \\
\hline
\end{tabular}

The following (Table 5) displays the Mean (SD), $N$ on each scale at the five different time points for each of the outcome measures used;

Table 5 - Outcome measure results at the five different timepoints. 


\begin{tabular}{|l|l|l|}
\hline Time point & Mean (SD) & $\boldsymbol{N}$ \\
\hline 1 & $40.83(9.77)$ & 60 \\
2 & $44.35(8.22)$ & 60 \\
3 & $45.85(8.63)$ & 60 \\
4 & $47.47(8.14)$ & 60 \\
5 & $49.42(9.45)$ & 60 \\
\hline \multicolumn{3}{|l|}{} \\
\hline \multicolumn{3}{|l|}{ Burden Assessment Scale } \\
\hline Time point & Mean (SD) & $\boldsymbol{N}$ \\
\hline 1 & $46.08(11.60)$ & 60 \\
2 & $39.63(12.19)$ & 60 \\
3 & $37.63(10.83)$ & 60 \\
4 & $36.48(11.31)$ & 60 \\
5 & $34.28(10.45)$ & 60 \\
\hline \multicolumn{3}{|l|}{} \\
\hline Family Empowerment Scale \\
\hline Time point & Mean (SD) & $\boldsymbol{N}$ \\
\hline 1 & $80.20(19.49)$ & 60 \\
2 & $85.15(20.02)$ & 60 \\
3 & $88.63(20.21)$ & 60 \\
4 & $90.28(20.74)$ & 60 \\
5 & $96.17(19.61)$ & 60 \\
\hline
\end{tabular}

$\underline{\text { Inferential Statistics }}$

A one-way repeated measures ANOVA was used to ascertain if there was a significant difference somewhere among the scores on each scale between the five different time points. The ANOVA revealed that the mean scores on the Mental Health and Wellbeing Scale were statistically significantly different $(F(3.167$, $186.846)=21.840, p<0.05$ ). In relation to the Burden Assessment Scale there was also a statistically significant difference $(F(3.214,189.654=25.734, p<0.05)$. The mean scores on the Family Empowerment Scale were also statistically significantly different $(F(2.633,155.363=16.998, p<0.05)$.

ANOVA however does not tell us between which specific time points the differences in scores were significant, just that there was an overall variance. In order to identify which means differed on each scale and at what time points, it is necessary to conduct further post-hoc tests. To ascertain where the specific significant differences occurred, a series of Bonferroni post hoc tests were performed, which allows us to make comparisons as to which of the mean scores differed between the five different time points. 
Table 6 below show the $p$ values generated from the post-hoc tests and indicate if there was a significant difference in individual's mean scores between each of the five time points on each of the scales. ( ${ }^{*}$ denotes significant at the $<0.05$ level)

\section{Table 6 - Mental Health and Wellbeing Scale}

\begin{tabular}{llllll}
\hline $\begin{array}{l}\text { Time } \\
\text { point }\end{array}$ & 1 & 2 & 3 & 4 & 5 \\
\hline 1 & - & $0.03^{*}$ & $0.00^{*}$ & $0.00^{*}$ & $0.00^{*}$ \\
2 & - & - & .928 & $0.02^{*}$ & $0.00^{*}$ \\
3 & - & - & - & 0.80 & $0.00^{*}$ \\
4 & - & - & - & - & 0.09 \\
\hline
\end{tabular}

\section{Burden Assessment Scale}

\begin{tabular}{llllll}
\hline $\begin{array}{l}\text { Time } \\
\text { point }\end{array}$ & 1 & 2 & 3 & 4 & 5 \\
\hline 1 & - & $0.00^{*}$ & $0.00^{*}$ & $0.00^{*}$ & $0.00^{*}$ \\
2 & - & - & 1.00 & 0.09 & $0.00^{*}$ \\
3 & - & - & - & 1.00 & $0.00^{*}$ \\
4 & - & - & - & - & $0.02^{*}$ \\
\hline
\end{tabular}

\section{Family Empowerment Scale}

\begin{tabular}{llllll}
\hline $\begin{array}{l}\text { Time } \\
\text { point }\end{array}$ & 1 & 2 & 3 & 4 & 5 \\
\hline 1 & - & 0.08 & $0.03^{*}$ & $0.03^{*}$ & $0.00^{*}$ \\
2 & - & - & 1.00 & 0.25 & $0.00^{*}$ \\
3 & - & - & - & 1.00 & $0.00^{*}$ \\
4 & - & - & - & - & $0.00^{*}$ \\
\hline
\end{tabular}

From the post hoc tests in relation to each scale the mean scores increased significantly between the time points indicated by the asterisks.

In relation to the Mental Health and Wellbeing Scale, although the scores increased between all the time points, 2-3, 3-4 and 4-5 were not significant. It is important to note however that the scores on the Mental Health and Wellbeing scale display that from time point 1 through to time point 5 the scores did increase at each point and were statistically significant. Why they did not increase between the other time 
points is unclear. But an increase in mental health and wellbeing is indicative of positive outcomes.

In relation to the Burden Assessment Scale, again, there was a decrease in the mean scores from time point 1 - time point 5 overall, which is indicative of a positive outcome and reduced burden. There was not a significant decrease in the mean scores from 2-3, 2-4 and 3-4.

On the family empowerment scale, again the significant increases indicated increased reports of being empowered hence a positive outcome. The asterisks indicate mean scores. Interesting that on this scale there is no significant increase in scores from 1-2. Also there is no significant increase from time 2-3, 2-4 and 3-4.

The overall message from the results is that the programme obviously had a positive impact on all three aspects that were measured. The means all fell in a positive direction, although a handful were close to but not statistically significantly different.

\section{Limitations}

This service evaluation study was conducted in one site and the site in which it was developed. The carer consultant who led this evaluation and development of the intervention was also the peer worker who delivered the interventions. What is not known is, are the results unique to the individual peer worker. The transferability of this programme and generalisability of the results should therefore be treated with caution and further replication of this model and its delivery in another site with different trainers, is therefore required. There were a disproportionate and increased number of carers who identified their family member as having borderline personality disorder and bipolar disorder, compared to other identified disorders. The results analysed include programme completers only, and therefore we do not establish or report why others dropped out after the mental health awareness stage or why a third of participants chose not to engage in the skills and problem-solving components. Hence the results presented within this paper may have been skewed, as it could be assumed that those who complete the programme were by virtue of their engagement inevitably going to report positive experiences. Conversely it could be argued non-completers or those who chose not to complete the full programme may have reported different experiences.

The use of a waiting list control group to provide comparisons of the effect of the intervention would have strengthened this study. If further replication studies testing out the effectiveness of this study were carried out, this should be considered and should therefore move beyond service evaluation into a powered research study. Any replication study also should include different trainers to establish if the carer who developed and delivered all training in this study is in fact the active ingredient 
or if the content of this intervention can be delivered with similar results by other carer peer support workers, hence displaying the transferability of the intervention.

\section{Discussion}

The positive findings from this service evaluation study can be seen to support the view that a psychoeducational programme is able to positively improve the mental wellbeing of carers and reduce carer burden, whilst also improving empowerment. The highly encouraging results from analysis of the three outcome measurements (mental health wellbeing, burden and empowerment) demonstrated that the programme had a positive impact from baseline to end measurement for carers of family members with mental health difficulties. The results also suggested that the programme had a positive, incremental effect across each of the five, measured time points. The post-programme support that is offered to participants could also ensure that the impact of the programme is continuous and that improvements in participant's mental health wellbeing, reduced burden and enriched empowerment are not lost once the programme has ended.

This is the first evaluation of the impact of a carer led psychoeducation intervention for carers of people with mental health difficulties in secondary mental health services. The evidence from this study indicates that carers will engage with and are able to attend a carer peer led psychoeducational programme. The number of referrals from the programme also indicates that there is significant demand for such a service. Implementation of the programme is relatively straight forward. The key challenges for practical implementation is have the right carer to lead and deliver the programme, the right support system in place for them (financial and supervision).

The results indicate that although almost half did not engage or only partially engaged with the programme. A third of participants who partially completed the programme leaving post psychoeducation group indicates that understanding of their family members presenting condition is of great importance. However, we did not explore why they chose to discontinue the programme prematurely. A larger proportion however completed the full programme and therefore are indicative of carer commitment to learn not just about the mental health problems but also to explore their own skills to support and coping mechanism skills development. It could however be argued that those who completed and who's results are analysed here may have provided skewed data. As by completing the programme this could be indicative of satisfaction with the programme.

This model of carer peer psychoeducation programme does provide evidence that with the correct structures and supportive systems in place, a co-delivered but peer led carer initiative in mental health carer programmes should be embraced and further developed and researched. The role of the peer carer roles however required not just supportive and supervisory practice but also investment to ensure that 
financially these roles are embraced, funded and supported giving meaningful and paid opportunities for people with lived experience of caring to share their expertise and provide support to other people with similar experiences.

What we do not know, as there was no follow up is the durability of the outcomes reported. Training evaluations have indicated that once complete the effectiveness can decline and that learning during the intervention or learning phase is always going to show some impact (Health Foundation, 2012). The measures however were not measuring knowledge generation but instead were measures of wellbeing, burden and empowerment. We also do not know if this programme and its impact is due to the delivery team, who also developed this programme and hence have had a unique impact or whether the programme is transferable and has potential to show similar results if delivered in a different locality with a different training team. It is anticipated that a further evaluation and sharing of this programme in a different area will be performed to establish its potential transferability and generalisability of the findings reported here. Very low numbers of people with family members with schizophrenia engaged in this training and the reasons for this unclear.

\section{Conclusion}

This is the first evaluation of the impact of a carer led psychoeducation intervention for carers of people with mental health difficulties in secondary mental health service and the positive results from the analysis of the three outcomes of improved mental wellbeing, reduced burden and enriched empowerment indicate that peer carer supported opportunities should be encouraged and supported. This project has identified that peer developed and delivered programmes are well received by carers of people with mental health difficulties. That psychoeducational component is in highest demand and additional skills development are also welcomed and embraced by carers of people with mental health difficulties. The peer carer delivering and leading this process has been well received and this innovative programme has displayed promising outcomes. The challenge now is in being able to further replicate this in another locality, to establish if it's the training programme, the model of delivery or the specific delivery contributors that have been responsible for the results indicated. Implementation of the programme is relatively straightforward. The key challenges for practical implementation is have the right carer to lead and deliver the programme, the right support system in place for them financially and with supervision.

\section{Key components for the replication of this project}

1. A Peer Worker (Carer background) will need to be identified alongside occupational expertise to support and deliver the programme in coproduction however with the peer worker taking a non-tokenistic and leading role. Support structures and institutional buy in will be required. 
2. There will be a requirement for support or time factored into the peer workers role to ensure the necessary administration duties are complete within the programme.

3. We strongly advocate that peer support workers should be valued and paid roles. Hence the development of a clear job description and personal specification for the training role is required.

4. Time will be required to develop an effective recruitment campaign, this may be via advertising and raising the profile of the project for carers. However furthermore the team supporting this project should ensure they also attend team meetings and spread the word to the practitioners on the front line of clinical practice.

5. Ongoing supervision of the peer worker by professionals supporting the project will be required.

6. Anyone interested in find out more about this initiative should make contact with the corresponding author who is willing to discuss and keen to provide train the trainer type opportunities for those wishing to replicate this work.

\section{References}

Barrowclough, C and Tarrier, N (1997) Families of Schizophrenic Patients, Cognitive Behavioural Intervention. Cheltenham: Nelson Thornes.

Buteau, E., Dawkins, K \& Hoffman, P (2008) In Their Own Words Improving Services and Hopefulness for Families Dealing with BPD. Social Work in Mental Health. 6 (1/2), 203-214.

Cassidy, E., Hill, S. \& O'Callaghan, E. European Psychiatry. The Journal of the Association of European Psychiatrists. 16(8),446-450.

Cuijpers P. The effect of Family Interventions on Relatives Burden. Journal of Mental Health. 8 (3): 275-285.

Gunderson, J.G. \& Hoffman, P,D (2005) Understanding and treating borderline personality disorder - a guide for professionals and families. New York: American Psychiatric Association Publishing.

Health Foundation (2012) Evidence Scan, Quality Improvement Training for Healthcare Professionals.

https://www.health.org.uk/sites/health/files/QualitylmprovementTrainingForHealthcar eProfessionals.pdf [Accessed 04/10/2018].

Hoffman \& Fruzzetti (2005) National Education Alliance for BPD Family Connections: A Program for Relatives of Persons with Borderline Personality Disorder Family Process, Vol,44 NO.2, 2005 @ FPI, INC. 
Kageyama, M., Nakamura, Y., Kobayashi, S \& Yokoyama, K (2016) Validity and reliability of the Family Empowerment Scale for caregivers of adults with mental health issue. Journal of Psychiatric Mental Health Nursing. 23 (8) 521-531.

Kilpatrick,E.,Keeney,S.,McCauley C.O (2017)Tokenistic or genuinely effective? Exploring the views of voluntary sector staff regarding the emerging peer support worker role in mental health. Journal Psychiatric Mental Health Nursing .24:503-512

Kirtley, J., Chiocchi, J., Cole, J., Sampson, M (2018) Stigma, emotional appraisal and the family environment as predictors of carer burden for relatives of individuals who meet the diagnostic criteria for borderline personality disorder. Personality and Mental Health Journal of Personality Disorders, 31, 1-19.

McFarlane, W, R., Dixon, L., Lukens, E \& Lucksted, A (2003) Family

Psychoeducation (FPE) Journal of Marital \& Family Therapy. 29 (2) 223-45.

McFarlane W, R., Dixon, L., Lukens, E \& Lucksted A (2012) Recent

Developments in Family Psychoeducation as an; Evidence Based Practice. Journal of Marital \& Family Therapy. (1) 101-21.

Motlova, L., Dragomirecka, E., Spaniel, F., Goppoldova, E \& Zalesky, R. (2006) Relapse Prevention does group Family Psychoeducation Matter. International Journal of Psychiatry in Clinical Practice. 10 (1) 38-44.

Mottaghipour, Y \& Bickerton, A (2005) The Pyramid of Family Care: A framework for family involvement with adult mental health services, Australian e-Journal for the Advancement of Mental Health, 4:3, 210-217, DOI: 10.5172/jamh.4.3.210.

National Institute for Mental Health in England (NIMHE) (2003a) Personality Disorder: No longer a diagnosis of exclusion, policy implementation guidance for the development of services for people with personality disorder. NIMHE: London.

National Institute for Mental Health in England (NIMHE) (2003b) Breaking the Cycle of Rejection: The Personality Disorder Capabilities Framework. NIMHE, London.

NICE Quality Standard 88 (2015) Personality disorders: borderline and antisocial. https://www.nice.org.uk/guidance/QS88 [Accessed 04/10/2018].

Pilling, S., Bebbington, P., Kuipers, E., Garety, P., Geddes, J., Orbach, G., Morgan, C (2002) Psychological treatments in schizophrenia: I. Meta-analysis of family intervention and cognitive behaviour therapy. Psychological Medicine. 32 (5): 763-782.

Reinhard, S. C., Gubman, G. D., Horwitz, A. V., \& Minsky, S (1994). Burden Assessment Scale for families of the seriously mentally ill. Evaluation and Program Planning, 17(3), 261-269. 
Shah AJ, Wadoo O, Latoo J (2010) Psychological Distress in Carers of People with Mental Disorders. British Journal of Medical Practitioners. 3(3): a327. http://www.bjmp.org/content/psychological-distress-carers-people-mental-disorders [Accessed 04/10/2018].

Sin, J., Gillard, S., Spain,D., Cornelius, V., Chen, T \& Henderson, C (2017) Effectiveness of psychoeducational interventions for family carers of people with psychosis: A systematic review and meta-analysis.

Tennant, R ., Hiller, L., Fishwick, R., Platt, S., Joseph, S., Weich, S., \& StewartBrown, $\mathbf{S}$ (2007). The Warwick-Edinburgh mental well-being scale (WEMWBS): development and UK validation. Health and Quality of Life Outcomes. 5(1), 63. 